\title{
The Notion of Stability Defect in Game Control Problems
}

\author{
VLADIMIR N. USHAKOV \\ Institute of Mathematics \\ and Mechanics
}

ul. S. Kovalevskoi 16, Ekaterinburg RUSSIA 620219

\author{
SERGEY A. BRYKALOV \\ Institute of Mathematics \\ and Mechanics
}

ul. S. Kovalevskoi 16, Ekaterinburg

RUSSIA 620219

\author{
YAROSLAV A. LATUSHKIN \\ Institute of Mathematics \\ and Mechanics
}

ul. S. Kovalevskoi 16, Ekaterinburg

RUSSIA 620219

\begin{abstract}
The stability property in a game problem of the approach of a conflict-controlled system to a target set at a fixed terminal moment is investigated. The notion of a stability defect is introduced for sets in the space of game positions.
\end{abstract}

Key-Words: Differential games, Control theory

\section{Introduction}

A conflict-controlled system on a finite time interval is studied. Issues related to the stability property, which is one of the central notions in the theory of positional differential games, are investigated. In the end of the 1960s (see [1-4]), the stability property was introduced and used as the basis for the notion of stable bridge and extremal shift to the stable bridge. In the first publications, the stability property was defined in terms of controls of antagonistic players.

In the following decades, there has been an evolution in the description of this property.

In [5-8] stability is represented as the property of the weak invariance of a set in the position space with respect to a family of differential inclusions related to the dynamics of a conflict-controlled system. These differential inclusions contain the control vector of the second player as a parameter.

In the first half of the 1970s, stability-based constructions were used for studying new problems, in particular, the game problem of pursuit-evasion with functional target set [9], as well as the guaranteed control problem with incomplete information about the state of a controlled object $[10,11]$.

In the middle of the 1970s, a new definition of stability emerged and gradually a new direction based on this definition was formed, namely, unification of differential games. In Krasovskii's papers [12, 13], a definition of unification models was given, their properties were studied, and possibilities of their application in various dynamics game problems were specified. The essence of unification is that the stability property is formulated in terms of vectors of conjugate variables and the Hamiltonian of a conflict-controlled system. Under unification, the structure of a conflict- controlled system - its right-hand side - becomes insignificant, and the system's Hamiltonian acquires importance. From the viewpoint of the following years, it becomes clear that one of the most important features of unification is that the stability property is expressed in the form that conforms to constructions of nonsmooth and convex analysis. Unification is also important for comparing conflict-controlled systems. For example, the fact that two systems with identical Hamiltonians are equivalent with respect to the solution of a differential game becomes absolutely clear. Some aspects of unification are also considered in [14, $15]$.

In the second half of the 1970s and in the beginning of the 1980s, investigations related to constructing stable bridges in game problems were conducted in several directions. In [16-18] the programmed iteration method for calculating the game value function and stable bridges in differential games was suggested. Methods and algorithms for approximate calculation of stable bridges were actively developed [19-21]; for this purpose, it was very important to choose a suitable representation for the stability property, which made the computations much easier.

The next stage concerning the representation of the stability property dates back to the beginning of the 1980s. By this time, several concrete differential games had been considered, in which the stability property assumed formulations not conforming to traditional schemes. The stability property for these games could be expressed by means of only a finite number of differential inclusions.

Thus, by the beginning of the 1980s, there had been several representations for describing the stability property, which is very important in differential games. Though these representations have different 
forms, they single out the same bridges, and therefore they are equivalent in essence.

In the first half of the 1980 s, a rather general formulation of the stability property emerged [22, 23]. It absorbed some of the known formulations, including those which did not comply with traditional schemes. In this formulation, just as in the unification scheme $[12,13]$, the Hamiltonian of a conflict-controlled system is present explicitly.

Then, in the middle of the 1980s, an infinitesimal representation of the stability property was obtained [24]. This representation was expressed in terms of Bouligand cones or right derivatives of a corresponding set. As Subbotin [25] has shown, this representation is useful not only when considering theoretical issues of differential games, but also for developing the theory of generalized (minimax and viscosity) solutions of Hamilton-Jacobi equations. Infinitesimal solutions related to Bouligand cones were later applied for studying more general first-order partial differential equations [26].

In the present paper we show that it is convenient to use constructions involved in the infinitesimal representation of the stability property to broaden the notion of stability. This leads to the expansion of the sphere of applicability of the extremal shift method.

\section{Statement of the Conflict Control Problem}

Let the behavior of a conflict-controlled system on the interval $\left[t_{0}, \vartheta\right], t_{0}<\vartheta<\infty$, be described by the system

$$
\dot{x}=f(t, x, u, v), x\left(t_{0}\right)=x_{0}, u \in P, v \in Q .
$$

Here, $x \in R^{m}$ is the phase vector of the system, $u$ and $v$ are controls of the first and second players, and $P$ and $Q$ are compact sets in the spaces $R^{p}$ and $R^{q}$, respectively. The symbol $R^{n}$ denotes the $n$-dimensional Euclidean space.

Assume that the following conditions are satisfied:

A. The vector function $f(t, x, u, v)$ is defined and continuous in variables $(t, x, u, v)$ on $\left[t_{0}, \vartheta\right] \times R^{m} \times$ $P \times Q$, and for any compact set $D \subset\left[t_{0}, \vartheta\right] \times R^{m}$ there exists $L=L(D) \in(0, \infty)$ such that

$$
\begin{gathered}
\left\|f\left(t, x^{(1)}, u, v\right)-f\left(t, x^{(2)}, u, v\right)\right\| \leq \\
L\left\|x^{(1)}-x^{(2)}\right\|
\end{gathered}
$$

for all $\left(t, x^{(i)}, u, v\right) \in D \times P \times Q, \quad i=1,2$.
B. There exists $\mu \in(0, \infty)$ such that $\|f(t, x, u, v)\| \leq \mu(1+\|x\|)$ for all $(t, x, u, v) \in$ $\left[t_{0}, \vartheta\right] \times R^{m} \times P \times Q$.

Here $\|f\|$ is the norm of a vector $f$ in the corresponding Euclidean space.

The differential game under consideration is antagonistic and consists of two problems - an approach problem and an evasion problem [8]. In the approach problem facing the first player, it is required to secure that the motion $x(t), t \in\left[t_{0}, \vartheta\right]$, of system (1) gets at the moment $\vartheta$ to a given compact set $M$ in $R^{m}$, whichever admissible control the second player selects. It is required to solve the problem in the class of positional procedures of control with a guide of the first player [8].

The evasion problem facing the second player consists in securing that the motion $x(t), t \in\left[t_{0}, \vartheta\right]$, of system (1) at the moment $\vartheta$ evades some closed $\varepsilon$ neighborhood $M_{\varepsilon}$ of the compact set $M$ for all admissible controls of the second player. It is required to solve the problem in the class of counterpositional procedures of control with a guide of the second player [8].

The following alternative [8] holds for the formulated problem: there exists a closed set $W^{0} \subset\left[t_{0}, \vartheta\right] \times$ $R^{m}$ (the maximal $u$-stable bridge) such that for all initial positions $\left(t_{*}, x_{*}\right) \in W^{0}$ the approach problem is solvable and for all initial positions $\left(t_{*}, x_{*}\right) \in$ $\left(\left[t_{0}, \vartheta\right] \times R^{m}\right) \backslash W^{0}$ the evasion problem is solvable.

According to the extremal shift principle [8], the resolving control procedure of the first player can be implemented for initial positions $\left(t_{*}, x_{*}\right) \in W^{0}$ as a positional control procedure with a guide, which aims the motion $x(t)$ of controlled system (1) at the motion of a guide through the bridge $W^{0}$. As is known, the main difficulty of the approach problem falls at finding the bridge $W^{0}$ in the position space $\left(t_{*}, x_{*}\right)$ of the differential game.

The problem of finding $W^{0}$ in the position space is one of the most difficult problems in constructing solutions of a differential game. The definition of the bridge $W^{0}$ is not an immediate recipe for finding it. In order to calculate $W^{0}$, one needs its analytical description, which, however, is possible only in rare cases. Therefore, it is important to develop methods and algorithms for the approximate construction of the bridge $W^{0}$. Such algorithms have been developed for some classes of problems [20, 21, 23].

As a result of approximate construction, we obtain not the bridge $W^{0}$, but some other set in $\left[t_{0}, \vartheta\right] \times$ $R^{m}$, which we denote by $\mathcal{W}^{0}$. The set $\mathcal{W}^{0}$ satisfies the boundary condition $\mathcal{W}^{0}(\vartheta)=M$, where $\mathcal{W}^{0}(t)=\left\{x \in R^{m}:(t, x) \in \mathcal{W}^{0}\right\}$.

Generally speaking, for positions $\left(t_{*}, x_{*}\right) \in \mathcal{W}^{0}$, not the initial problem of approaching $M$, but the less 
stringent problem of approaching some $\varepsilon$ neighborhood $M_{\varepsilon}$ of the set $M$ is solvable. In this case, to construct the first player's control guaranteeing that the motion $x(t)$ of system (1) gets to $M_{\varepsilon}$, we use a positional procedure aiming the motion $x(t)$ at some broken line going through $\mathcal{W}^{0}$ and hitting the set $\mathcal{W}^{0}(\vartheta)=M$ at the final moment $\vartheta$. This broken line can be interpreted as a guide's motion.

In the following sections of this work, we consider a bounded closed set $W^{*}$ from $\left[t_{0}, \vartheta\right] \times R^{m}$, $W^{*}(\vartheta)=M$, assuming that it satisfies some conditions (see Section 4). For $W^{*}$ and the positions $\left(t_{*}, x_{*}\right) \in W^{*}$, we define a control procedure similar to the above-mentioned procedure for the set $\mathcal{W}^{0}$. This procedure is in essence a procedure of control with a guide, and we call it for short a $W^{*}$ control procedure of the first player. In Section 5 we neatly estimate the $\varepsilon$ neighborhood of the set $M$ to which all motions $x(t)$ from points $\left(t_{*}, x_{*}\right) \in W^{*}$ can be brought by means of the $W^{*}$ control procedure.

\section{Stability of Sets in the Space of Game Positions}

The maximal stable bridge $W^{0}$ consists of all $\left(t_{*}, x_{*}\right) \in\left[t_{0}, \vartheta\right] \times R^{m}$ from which the approach problem is solvable. Using this fact and Condition $\mathrm{B}$, we can choose in $\left[t_{0}, \vartheta\right] \times R^{m}$ a sufficiently large closed domain $D$ containing the bridge $W^{0}$ and all the motions from a sufficiently small neighborhood of $W^{0}$.

However, in the subsequent sections we will consider sets $W^{*} \subset\left[t_{0}, \vartheta\right] \times R^{m}$, which are not necessarily stable bridges and which may differ substantially from $W^{0}$, so the above-mentioned choice of the domain $D$ is insufficient for us.

Let us consider a compact set $W^{*} \subset\left[t_{0}, \vartheta\right] \times R^{m}$, $W^{*}(\vartheta) \subset M$, whose properties will be detailed later, and refine the choice of the domain $D$.

Denote by $h\left(W_{2}, W_{1}\right)$ the Hausdorff deviation of the set $W_{2}$ from $W_{1}$, where $W_{1}$ and $W_{2}$ are from $R^{m}$.

Let a number $\varepsilon_{*}>0$ satisfy the following inequalities:

$$
\begin{gathered}
\varepsilon_{*}>\sup _{t \in\left[t_{0}, \vartheta\right]} h\left(W^{*}(t),\{\boldsymbol{0}\}\right), \\
\varepsilon_{*}>\rho_{*}+\mu\left(\vartheta-t_{0}\right) e^{\mu\left(\vartheta-t_{0}\right)}
\end{gathered}
$$

Here $\mathbf{0}$ is the zero in $R^{m},\{\boldsymbol{0}\}$ is the set consisting of the zero, $\rho_{*}=h(M,\{\mathbf{0}\})$.

Then the cylinder

$$
Z=\left\{(t, x): t \in\left[t_{0}, \vartheta\right],\|x\| \leq \varepsilon_{*}\right\}
$$

in $\left[t_{0}, \vartheta\right] \times R^{m}$ contains both $W^{*}$ and $W^{0}$. domain

The cylinder $Z$ is contained in the bounded closed

$D=\left\{(t, x): t \in\left[t_{0}, \vartheta\right],\|x\| \leq\left(\varepsilon_{*}+\mu\left(t-t_{0}\right)\right) e^{\mu\left(t-t_{0}\right)}\right\}$

from $\left[t_{0}, \vartheta\right] \times R^{m}$.

The domain $D$ is an integral funnel on $\left[t_{0}, \vartheta\right]$ of the differential equation

$$
\dot{x} \in U(x)=\left\{f \in R^{m}:\|f\| \leq \mu(1+\|x\|)\right\}
$$

with the initial set

$$
D\left(t_{0}\right)=\left\{x^{0} \in R^{m}:\left\|x^{0}\right\| \leq \varepsilon_{*}\right\} .
$$

Let $G$ be the largest of balls $U(x), \quad(t, x) \in D$, and let $\rho$ be its radius.

We have the inclusion

$$
F(t, x) \subset U(x), \quad(t, x) \in\left[t_{0}, \vartheta\right] \times R^{m},
$$

and, therefore, all solutions of the differential inclusion

$$
\dot{x} \in F(t, x), \quad\left(t_{*}, x\left(t_{*}\right)\right)=\left(t_{*}, x_{*}\right) \in Z
$$

satisfy the inclusion $F(t, x) \subset G, \quad(t, x) \in D$.

Assume

$$
\begin{gathered}
\Pi_{l}(t, x)=\left\{f \in R^{m}:\langle l, f\rangle \leq H(t, x, l)\right\}, \\
F_{l}(t, x)=F(t, x) \bigcap \Pi_{l}(t, x), \\
(t, x, l) \in D \times S, \quad S=\left\{l \in R^{m}:\|l\|=1\right\} .
\end{gathered}
$$

We have the inclusion

$$
F_{l}(t, x) \subset G, \quad(t, x, l) \in D \times S .
$$

Now we formulate the definitions of a stable bridge $W$ and a maximal stable bridge $W^{0}$ in terms of a family $\mathcal{L}=\left\{(t, x) \mapsto F_{l}(t, x), l \in S\right\}$ of mappings $(t, x) \mapsto F_{l}(t, x), \quad(t, x) \in D$, corresponding to the vectors $l \in S$.

These definitions are preceded by the definition of a stable absorption operator.

Namely, denote by $X_{l}\left(t^{*} ; t_{*}, x_{*}\right)$ the set of all $x^{*} \in R^{m}$ satisfying the equality $x\left(t^{*}\right)=x^{*}$, where $x(\cdot)=\left\{x(t): t_{*} \leq t \leq t^{*}\right\}$ is a solution of the differential inclusion

$$
\dot{x} \in F_{l}(t, x), \quad x\left(t_{*}\right)=x_{*}, \quad t \in\left[t_{*}, t^{*}\right]
$$

$X_{l}^{-1}\left(t_{*} ; t^{*}, X^{*}\right)=\left\{x_{*} \in R^{m}: X_{l}\left(t^{*} ; t_{*}, x_{*}\right) \bigcap X^{*} \neq \emptyset\right\}$,

$X^{*}$ is a set from $R^{m}$. 
Definition 1 The stable absorption operator $\pi$ in the approach problem is the mapping $\left(t_{*} ; t^{*}, X^{*}\right) \mapsto 2^{R^{m}}$ given by

$$
\begin{gathered}
\pi\left(t_{*} ; t^{*}, X^{*}\right)=\bigcap_{l \in S} X_{l}^{-1}\left(t_{*} ; t^{*}, X^{*}\right), \\
\left(t_{*}, t^{*}, X^{*}\right) \in \Delta \times 2^{R^{m}} .
\end{gathered}
$$

Here, $\Delta=\left\{\left(t_{*}, t^{*}\right): t_{0} \leq t_{*}<t^{*} \leq \vartheta\right\}$.

Definition 2 A closed set $W \subset D$ is a u-stable bridge if

$$
\begin{gathered}
W(\vartheta) \subset M \\
W\left(t_{*}\right) \subset \pi\left(t_{*} ; t^{*}, W\left(t^{*}\right)\right), \quad\left(t_{*}, t^{*}\right) \in \Delta .
\end{gathered}
$$

Let $W^{0}$ be the union of all $u$-stable bridges $W \subset$ $D$. Then $W^{0}$ is the maximal (with respect to inclusion) $u$-stable bridge and is the positional absorption set in the approach problem under consideration (see [8]).

Recall also the infinitesimal formulation of the stability property expressed in terms of the family $\mathcal{L}$ (see [24]). Let us present this formulation in the form of a theorem.

Theorem 3 A nonempty closed set $W \subset D$ is a $u$ stable bridge if and only if

(1) $W(\vartheta) \subset M$;

(2) $\vec{D} W(t, x) \cap F_{l}(t, x) \neq \emptyset, t \in\left[t_{0}, \vartheta\right)$, $(t, x, l) \in W \times S$.

Here, $\vec{D} W(t, x)=\left\{d \in R^{m}: \quad d=\right.$ $\lim _{k \rightarrow \infty} \frac{w_{k}-x}{t_{k}-t}, \quad\left(t_{k}, w_{k}\right) \in W(k=1,2) \ldots ; t_{k} \downarrow$ t as $\left.k \rightarrow \infty, \lim _{k \rightarrow \infty}\left(t_{k}, w_{k}\right)=(t, x)\right\}$.

It is evident that for the bridge $W^{0}$ Condition (1) of Theorem 1 takes the form $W^{0}(\vartheta)=M$. In addition, the bridge $W^{0}$ possesses the $T$ property: from $t_{0} \leq t_{*}<t^{*} \leq \vartheta$ and $W^{0}\left(t_{*}\right) \neq \phi$ it follows that $W^{0}\left(t^{*}\right) \neq \phi$. The $T$ property of the bridge $W^{0}$ can be characterized as the continuity property of $W^{0}$ as the time $t$ grows on $\left[t_{0}, \vartheta\right]$.

Further, we have

$$
X_{l}\left(t^{*} ; t_{*}, x_{*}\right) \bigcap W^{0}\left(t^{*}\right) \neq \phi,\left(t_{*}, x_{*}, l\right) \in W^{0} \times S,
$$

and, therefore, taking into account $X_{l}\left(t^{*} ; t_{*}, x_{*}\right) \subset$ $O_{\left(t^{*}-t_{*}\right) \rho}\left(x_{*}\right)$, we obtain

$$
W^{0}\left(t^{*}\right) \bigcap O_{\left(t^{*}-t_{*}\right) \rho}\left(x_{*}\right) \neq \phi, \quad\left(t_{*}, x_{*}\right) \in W^{0} .
$$

Here, $O_{\left(t^{*}-t_{*}\right) \rho}\left(x_{*}\right)=\left\{w \in R^{m}:\left\|w-x_{*}\right\| \leq\right.$ $\left.\rho\left(t^{*}-t_{*}\right)\right\}$.

Hence,

$$
\vec{D} W^{0}\left(t_{*}, x_{*}\right) \bigcap G \neq \phi
$$

for all $\left(t_{*}, x_{*}\right) \in W^{0}, t_{*} \in\left[t_{0}, \vartheta\right)$.

\section{The Stability Defect of Sets in the Position Space of the Game}

In this section we define the stability defect of a set contained in $D$.

Assume that the set $W^{*} \subset D$ from Section 3 satisfies the condition $W^{*}(\vartheta)=M$ and possesses the $T$ property.

Moreover, as a way of strengthening the $T$ property of the set $W^{*}$, we assume that the following condition is valid:

C. $W^{*}\left(t^{*}\right) \bigcap O_{\left(t^{*}-t_{*}\right) \rho}\left(x_{*}\right) \neq \emptyset,\left(t_{*}, x_{*}\right) \in W^{*}$, $t_{0} \leq t_{*}<t^{*} \leq \vartheta$.

Remark 4 Condition $\mathrm{C}$ means that the multivalued mapping $t \mapsto W^{*}(t), \quad t \in\left[t_{0}, \vartheta\right]$ changes (in some sense) not very fast at the points $\left(t_{*}, x_{*}\right) \in W^{*}, t_{*} \in$ $\left[t_{0}, \vartheta\right)$, as $t$ grows.

It follows from Condition $\mathrm{C}$ that

$\vec{D} W^{*}\left(t_{*}, x_{*}\right) \bigcap G \neq \emptyset,\left(t_{*}, x_{*}\right) \in \partial W^{*}, t_{*} \in\left[t_{0}, \vartheta\right) ;$ here, $\partial W^{*}$ is the boundary of the set $W^{*}$ in $\left[t_{0}, \vartheta\right] \times$ $R^{m}$.

Let us assign to each point $\left(t_{*}, x_{*}\right) \in \partial W^{*}, t_{*} \in$ $\left[t_{0}, \vartheta\right)$, the number

$$
\varepsilon\left(t_{*}, x_{*}\right)=\sup _{l \in S} \rho\left(\vec{D} W^{*}\left(t_{*}, x_{*}\right), F_{l}\left(t_{*}, x_{*}\right)\right) \geq 0 .
$$

Here we denote

$$
\rho\left(D^{*}, F^{*}\right)=\inf _{(d, f) \in D^{*} \times F^{*}}\|d-f\|,
$$

where $D^{*}$ and $F^{*}$ are sets from $R^{m}$.

We call the value $\varepsilon\left(t_{*}, x_{*}\right)$ the stability defect of the set $W^{*}$ at the point $\left(t_{*}, x_{*}\right) \in \partial W^{*}, t_{*} \in\left[t_{0}, \vartheta\right)$.

Remark 5 Under some mild conditions on system (1), the supremum in the expression for $\varepsilon\left(t_{*}, x_{*}\right)$ is attained at all points $\left(t_{*}, x_{*}\right) \in \partial W^{*}, t_{*} \in\left[t_{0}, \vartheta\right)$. One of such conditions is, for example, the condition

$$
\begin{gathered}
H_{*}\left(t_{*}, x_{*}, l\right)<H\left(t_{*}, x_{*}, l\right)<H^{*}\left(t_{*}, x_{*}, l\right), \\
\left(t_{*}, x_{*}\right) \in \partial W^{*}, t_{*} \in\left[t_{0}, \vartheta\right), \quad l \in S ;
\end{gathered}
$$

here, $\quad H_{*}\left(t_{*}, x_{*}, l\right)=\min _{g \in F\left(t_{*}, x_{*}\right)}\langle l, g\rangle$, $H^{*}\left(t_{*}, x_{*}, l\right)=\max _{g \in F\left(t_{*}, x_{*}\right)}\langle l, g\rangle$. 
For subsequent considerations, it is convenient to replace the set $\vec{D} W^{*}\left(t_{*}, x_{*}\right)$ from the expression for $\varepsilon\left(t_{*}, x_{*}\right)$ by a smaller compact set; here the value $\varepsilon\left(t_{*}, x_{*}\right)$ is retained.

Consider the set

$$
\vec{D}^{\nabla} W^{*}\left(t_{*}, x_{*}\right)=\vec{D} W^{*}\left(t_{*}, x_{*}\right) \bigcap 3 G
$$

where $3 G=\{3 g: g \in G\}$.

Since $\vec{D} W^{*}\left(t_{*}, x_{*}\right) \cap G \neq \emptyset, \quad F_{l}\left(t_{*}, x_{*}\right) \subset G$ for $\left(t_{*}, x_{*}\right) \in \partial W^{*}, t_{*} \in\left[t_{0}, \vartheta\right), l \in S$, we have

$$
\begin{aligned}
& \rho\left(\vec{D}^{\nabla} W^{*}\left(t_{*}, x_{*}\right), F_{l}\left(t_{*}, x_{*}\right)\right) \\
& =\rho\left(\vec{D} W^{*}\left(t_{*}, x_{*}\right), F_{l}\left(t_{*}, x_{*}\right)\right)
\end{aligned}
$$

for $\left(t_{*}, x_{*}\right) \in \partial W^{*}, t_{*} \in\left[t_{0}, \vartheta\right), \quad l \in S$.

Hence we have the following representation:

$$
\begin{gathered}
\varepsilon\left(t_{*}, x_{*}\right)=\sup _{l \in S} \rho\left(\vec{D}^{\nabla} W^{*}\left(t_{*}, x_{*}\right), F_{l}\left(t_{*}, x_{*}\right)\right), \\
\left(t_{*}, x_{*}\right) \in \partial W^{*}, t_{*} \in\left[t_{0}, \vartheta\right) .
\end{gathered}
$$

Assume for $t_{*} \in\left[t_{0}, \vartheta\right)$

$$
\varepsilon\left(t_{*}\right)=\sup _{\left(t_{*}, x_{*}\right) \in \Lambda\left(t_{*}\right)} \varepsilon\left(t_{*}, x_{*}\right),
$$

where $\Lambda\left(t_{*}\right)=\partial W^{*} \bigcap \Gamma_{t_{*}}, \Gamma_{t_{*}}=\left\{(t, x): t=t_{*}\right\}$.

Let us call the value $\varepsilon\left(t_{*}\right)$ the stability defect of the set $W^{*}$ at the moment $t_{*} \in\left[t_{0}, \vartheta\right)$. At the same time, we have a nonnegative function $\varepsilon(t)$ on $\left[t_{0}, \vartheta\right)$, which can be interpreted as a characteristic of the instability extent of the set $W^{*}$. have

If $W^{*}$ is a $u$-stable bridge, then by Theorem 1 we

$$
\vec{D} W^{*}\left(t_{*}, x_{*}\right) \bigcap F_{l}\left(t_{*}, x_{*}\right) \neq \phi,
$$

$\left(t_{*}, x_{*}\right) \in \partial W^{*}, t_{*} \in\left[t_{0}, \vartheta\right), l \in S$; therefore,

$$
\vec{D}^{\nabla} W^{*}\left(t_{*}, x_{*}\right) \bigcap F_{l}\left(t_{*}, x_{*}\right) \neq \phi,
$$

$\left(t_{*}, x_{*}\right) \in \partial W^{*}, t_{*} \in\left[t_{0}, \vartheta\right), \quad l \in S$.

Hence, $\varepsilon\left(t_{*}, x_{*}\right)=0, \quad\left(t_{*}, x_{*}\right) \in \partial W^{*}, \quad t_{*} \in$ $\left[t_{0}, \vartheta\right)$, and then $\varepsilon(t)=0$ on $\left[t_{0}, \vartheta\right)$.

Further, from the equality $\varepsilon(t)=0$ on $\left[t_{0}, \vartheta\right)$, we obtain

$$
\vec{D}^{\nabla} W^{*}\left(t_{*}, x_{*}\right) \bigcap F_{l}\left(t_{*}, x_{*}\right) \neq \phi,
$$

$\left(t_{*}, x_{*}\right) \in \partial W^{*}, l \in S, t_{*} \in\left[t_{0}, \vartheta\right)$, i.e., $W^{*}$ is a $u$-stable bridge.

We have shown that the stability of the set $W^{*}$ is equivalent to the equality $\varepsilon(t)=0$ on $\left[t_{0}, \vartheta\right)$. Hence, in the case $\varepsilon(t)=0$ on $\left[t_{0}, \vartheta\right)$, the rule of extremal shift to a guide moving through $W^{*}$ guarantees that the motion $x(t)$ of system (1) gets to $M$ if $\left(t_{*}, x\left(t_{*}\right)\right)=\left(t_{*}, x_{*}\right) \in W^{*}$.
This suggests that if the function $\varepsilon(t)$ corresponding to the set $W^{*}$ is small on $\left[t_{0}, \vartheta\right)$, then the rule of the extremal aiming at a guide moving through $W^{*}$ guarantees that the motion $x(t)$ of system (1) gets to a small $\varepsilon$ neighborhood of the set $M$ at the moment $\vartheta$. In addition, it is intuitively obvious that $\varepsilon$ can be expressed by means of the integral $\int_{t_{0}}^{\vartheta} \varepsilon(t) d t$ (if this function is integrable on $\left[t_{0}, \vartheta\right]$ ).

In order to validate these assertions, we impose additional restrictions on $W^{*}$ and $\varepsilon(t),\left[t_{0}, \vartheta\right)$ :

D. There exists a scalar function $\varphi^{*}(\delta) \downarrow 0$ as $\delta \downarrow 0$ such that

$$
h\left(x_{*}+\delta \vec{D}^{\nabla} W^{*}\left(t_{*}, x_{*}\right), \quad W^{*}\left(t_{*}+\delta\right)\right) \leq \delta \varphi^{*}(\delta)
$$

for $t_{*} \in\left[t_{0}, \vartheta\right),\left(t_{*}, x_{*}\right) \in \partial W^{*}, \delta \in\left(0, \vartheta-t_{*}\right)$. $\left[t_{0}, \vartheta\right]$

E. The function $\varepsilon(t)$ is Riemann integrable on

Here we denote $x_{*}+\delta X_{*}=\left\{x_{*}+\delta f: f \in X_{*}\right\}$, where $X_{*}$ is a set from $R^{m}$.

In publications [1-8], the positional control of the first player is described based on the rule of the extremal shift of the motion $x(t)$ of system (1) to the $u$-stable bridge $W$. The same rule can be applied when instead of the $u$-stable bridge we have the set $W^{*}$. The positional strategy $U^{l}(t, x)$ of the first player based on the rule of extremal shift to $W^{*}$ will be called for short the $W^{*}$-strategy of the first player. The following statement is true:

Theorem 6 The motion $x(t)\left(x\left(t_{*}\right)=x_{*} \in W^{*}\left(t_{*}\right)\right)$ on $\left[t_{*}, \vartheta\right]$, generated by $W^{*}$-strategy of the first player satisfies the inclusion

$$
\begin{gathered}
x(\vartheta) \in M_{\varepsilon}, \\
\text { where } \varepsilon=\varepsilon_{W^{*}}=e^{L\left(\vartheta-t_{0}\right)} \int_{t_{0}}^{\vartheta} \varepsilon(\tau) d \tau .
\end{gathered}
$$

The number $\varepsilon=\varepsilon_{W^{*}}$ (3) can be considered as a measure of instability of the set $W^{*}$. It is natural to call it a stability defect of the set $W^{*}$.

\section{Acknowledgements}

This work was supported by the Russian Foundation for Basic Research (project nos. 05-01-00601a, 06-01-00436, 04-01-96099ural_a) and by the State Program for Support of Leading Scientific Schools (project NSh-8512.2006.1). 


\section{References:}

[1] N. N. Krasovskii, Izv. Akad. Nauk SSSR, Ser. Tekh. Kibernetika, No. 5, 3 (1969).

[2] N. N. Krasovskii and A. I. Subbotin, Dokl. Akad. Nauk SSSR 188, 745 (1969).

[3] N. N. Krasovskii, Prikl. Mat. Mekh. 34 (2), 197 (1970).

[4] N. N. Krasovskii, Game Problems on the Encounter of Motions (Nauka, Moscow, 1970) [in Russian].

[5] N. N. Krasovskii and A. I. Subbotin, Dokl. Akad. Nauk SSSR 190, 523 (1969).

[6] N. N. Krasovskii and A. I. Subbotin, Differnts. Uravn. 6, 579 (1970).

[7] N. N. Krasovskii and A. I. Subbotin, Prikl. Mat. Mekh. 37 (2), 197 (1973).

[8] N. N. Krasovskii and A. I. Subbotin, Positional Differential Games (Nauka, Moscow, 1974) [in Russian].

[9] A. V. Kryazhimskii and Yu. S. Osipov, Prikl. Mat. Mekh. 37 (1), 3 (1973).

[10] N. N. Subbotina and A. I. Subbotin, Izv. Akad. Nauk SSSR, Ser. Tekh. Kibernetika, No. 5, 14 (1977).

[11] N. N. Krasovskii and Yu. S. Osipov, Dokl. Akad. Nauk SSSR 215, 780 (1974).

[12] N. N. Krasovskii, Dokl. Akad. Nauk SSSR 226, 1260 (1976).

[13] N. N. Krasovskii, Trudy Inst. Mat. Mekh. Ural'sk. Nauchn. Tsentr Akad. Nauk SSSR, No. 24, 32 (1977).

[14] M. I. Alekseichik, in Mathematical Analysis and Its Applications (Rostovskii Gos. Univ., Rostovon-Don, 1975), Vol. 7, pp. 191-199.

[15] V. N. Ushakov, Izv. Akad. Nauk SSSR, Ser. Tekh. Kibernetika, No. 5, 29 (1980).

[16] A. G. Chentsov, Mat. Sb. 99 (3), 394 (1976).

[17] A. G. Chentsov, Dokl. Akad. Nauk SSSR 240, 796 (1978).

[18] A. I. Subbotin and A. G. Chentsov, Guarantee Optimization in Control Problems (Nauka, Moscow, 1981) [in Russian].

[19] Algorithms and Programs for Solving Linear Differential Games, Ed. by A. I. Subbotin and V. S. Patsko (Inst. Mat. Mekh. Ural'sk. Nauchn. Tsentr Akad. Nauk SSSR, Sverdlovsk, 1984) [in Russian].

[20] E. A. Isakova, G. V. Logunova, and V. S. Patsko, in Algorithms and Programs for Solving Linear Differential Games, Ed. by A. I. Subbotin and V. S. Patsko (Inst. Mat. Mekh. Ural'sk. Nauchn. Tsentr Akad. Nauk SSSR, Sverdlovsk, 1984), pp. 127-158 [in Russian].
[21] A. M. Taras'ev and V. N. Ushakov, in Investigation of Minimax Control Problems (Inst. Mat. Mekh. Ural'sk. Nauchn. Tsentr Akad. Nauk SSSR, Sverdlovsk, 1985), pp. 82-90 [in Russian].

[22] A. M. Taras'ev and V. N. Ushakov, On Constructing Stable Bridges in a Minimax Evasion Problem, Available from VINITI, 1983, Sverdlovsk, No. 2454-83.

[23] A. M. Taras'ev, V. N. Ushakov, and A. P. Khripunov, Prikl. Mat. Mekh. 51 (2), 216 (1987).

[24] H. G. Guseinov, A. I. Subbotin, and V. N. Ushakov, Problems Control Inform. Theory 14 (6), 405 (1985).

[25] A. I. Subbotin, Minimax Inequalities and Hamilton-Jacobi Equations (Nauka, Moscow, 1991) [in Russian].

[26] A. I. Subbotin, Generalized Solutions of FirstOrder PDEs. The Dynamical Optimization Perspective, System \& Control: Foundation \& Appl. (Birkhäuser, Boston, 1995). 